\title{
Les suaires de Michel Tournier et d'Orlan : quelques poses photographiques
}

\author{
Catherine Parayre \\ Université Brock
}

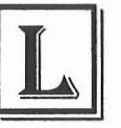

e suaire de Turin avec ses pâles marques, dont de nombreux croyants supposent que ce sont celles du corps de Jésus, est depuis 1578 un objet de vénération qui attire curieux et pèlerins. Il connut cependant un regain de popularité à partir de 1893, date des premières impressions photographiques du mystérieux linceul, lorsque celles-ci révélèrent l'image d'un corps " apparemment en trois dimensions " (Geimer 143) et suscitèrent divers échanges théologiques et scientifiques portant sur l'authenticité du suaire, les moyens techniques utilisés pour l'examiner ou encore le statut de l'image religieuse et des reliques dans la pensée collective. Sans doute de telles discussions sur le rôle de la photographie dans l'histoire du suaire renvoient-elles à un débat plus général ouvert par le constat que la photographie atteste le réel, qu'elle en témoigne et en donne la preuve. De nombreux écrits critiques abordent cette question essentielle dans l'art photographique, mais certains photographes en font également un thème de leurs créations. C'est, par exemple, le cas d'une nouvelle de Michel Tournier ${ }^{1}$, "Les suaires de Véronique ", et d'une série de photographies intitulée Saints-suaires de l'artiste Orlan, deux ouvres dans le domaine d'expression française qui font précisément du motif du suaire le symbole d'une réflexion complexe sur le portrait photographique, et ceci de manière étonnamment similaire, par exemple dans le but d'insister sur la surprise ou le choc que peut provoquer le spectacle photographique. À la question de savoir ce qu'apporte le motif du suaire à une semblable réflexion esthétique, une amorce de réponse se dessine dès lors qu'on prend en considération un événement attaché au culte du suaire de Turin. En effet, la cérémonie

1 Tournier est lui-même photographe. 
pendant laquelle celui-ci est montré aux fidèles s'appelle une ostentation. Or, rien n'est plus important pour Véronique et Orlan que d'exposer leurs suaires et de les offrir à l'appréciation des spectateurs. Outre cette dimension spectaculaire, on ne peut également guère ignorer l'aspect le plus troublant du suaire de Turin, à savoir que les empreintes qui y sont décelables, attribuent quasiment une corporalité à une figure absente. À Turin, ces singulières traces ont occasionné débats et polémiques, et même la plus haute autorité de l'Eglise, en la personne du pape Jean-Paul II, lors d'une visite dans la ville, a évité de prendre clairement position, invoquant le mystère et la force spirituelle dont la vénération du suaire témoigne, tout en se gardant d'affirmer l'authenticité du linceul (voir le discours de JeanPaul II tenu le 24 mai 1998). La présente étude argumentera que, dans la nouvelle de Tournier et dans le projet d'Orlan, l'étrange représentation du corps sur le suaire évoque un autre trouble, celui que Roland Barthes qualifie de "trouble de civilisation " apparu à l'invention de la photographie et par lequel il entend que celle-ci a radicalement modifié nos notions d'identité : "La photographie, c'est l'avènement de moi-même comme autre [...] La Photographie [transforme] le sujet en objet... Les autres - l'Autre - me désapproprient de moi-même, ils font de moi, avec férocité, un objet " (1116-1118). Selon Barthes, « ce trouble est au fond un trouble de propriété » $(1117)^{2}$. L'examen de différentes manifestations de ce trouble dans nos deux œuvres montrera que le motif du suaire y a pour fonction d'exhiber ce malaise jusqu'à l'excès de signes, nous rappelant, lecteurs et spectateurs que nous sommes, que la photographie, aussi quotidienne et banale qu'elle apparaît aujourd'hui, bouleverse néanmoins notre perception d'autrui et de nous-mêmes.

2 Tournier explore également ce thème dans La goutte d'or, lorsqu'un jeune Berbère prend peur de son portrait photographique : "La photographie constitue une sorte de double du visage réel. Ce double est dangereux aux yeux des Berbères musulmans qui le considèrent comme une partie intrinsèque de l'individu sur laquelle on peut agir pour le maléficier ", car la photographie permet à l'individu de " prendre conscience de lui-même dans le regard d'autrui ", regard qui pétrifie le sujet, en fixant l'image sur la pellicule, comme dans le mythe de la Méduse " qui pétrifie et tue ceux qui la regardent " (Bouloumié 145-146). 


\section{Photographies ostentatoires}

La nouvelle de Tournier met en scène une photographe, Véronique, qui a formé pour projet de rendre Hector, son compagnon et jeune modèle, plus photogénique que jamais et, à cet effet, pratique sur lui ce qu'elle nomme la "photographie directe » (169), c'est-à-dire qu'elle plonge directement le corps d'Hector dans le bain de révélateur, puis le couche sur le papier photographique. Férue de théorie, fascinée par la mort et les cadavres, Véronique conduit ses expériences avec autant d'audace que les médecins de la Renaissance qui pratiquaient des autopsies de plus en plus précises au grand dam de l'Eglise. L'histoire s'achève dans une galerie exposant les réalisations de la photographe regroupées sous le titre des "Suaires de Véronique ", lorsque le narrateur, qui compte celle-ci et Hector parmi ses connaissances, découvre, abasourdi, les toiles - ou suaires - fixées aux murs et les empreintes humaines qu'elles portent, et comprend soudainement que c'est le corps d'Hector qui en forme la matière.

Orlan, quant à elle, est célèbre pour son " art charnel ", à savoir les altérations chirurgicales auxquelles elle fait procéder sur son propre corps et qu'elle documente en direct, sur le plateau de l'opération, dans des films et dans des photographies. Son objectif consiste à mettre en scène la " défiguration et la refiguration " de sujets connus, par exemple la Vénus de Botticelli, la Joconde ou sainte Thérèse, dont les traits sont esquissés, mais aussi transformés dans les montages photographiques, aussi bien que dans la chair opérée de l'artiste (Orlan, Art charnel, 38). Les Saints-suaires réalisés dans les années 1990 représentent son visage, nettement visible, sous des morceaux de gaze utilisés pour absorber le sang et autres fluides lors de ces opérations et donnent l'impression que les traits de l'artiste y sont calqués, figés en une étrange pose. Le montage est bien réel : il la représente, tout en étant " fait d'elle-même " (Cros 154).

Bien que ces deux ouvres thématisent la mutilation et la souffrance du corps, il faut néanmoins se garder d'y voir uniquement, pour l'une, une intrigue psychologique et, pour l'autre, la mise en scène d'un questionnement existentiel. Certes, le suaire constitue la preuve de la mise à mort d'Hector perpétrée par Véronique afin de mettre en pratique certaines notions esthétiques et assouvir sa jalousie à l'égard de son amant, de même qu'il symbolise la mort à venir de tout être humain dans le travail d'Orlan. Toutefois, il est avéré dans la longue carrière de nos deux auteurs que ces derniers s'intéressent avant tout aux systèmes et structures de signification (Rosello 12 pour Tournier; Art charnel 38 pour Orlan), et si les personnages de Tournier « avancent au milieu d'une 'forêt de symboles' 
qui leur font signe " (Bouloumié 241), la même chose pourrait être dite d'Orlan. C'est ainsi que, dans "Les suaires de Véronique " et les Saintssuaires, ces signes renvoient lecteurs et spectateurs à la réflexion des deux auteurs sur la photographie et, en particulier, la pose photographique ${ }^{3}$. En fait, la nouvelle de Tournier et les commentaires d'Orlan sur les Saintssuaires forment un tissu dense d'exemples, de déclarations et de motifs récurrents précisant l'immobilité de la pose - le suaire est ainsi comparé à une statue, un masque, mais aussi un voile. Le suaire photographique est donc un tissu-théorie riche en allusions et en signes, à la fois pose photographique et réflexion sur celle-ci.

Le sujet est de taille. En effet, de l'avis de nombreux auteurs, la photographie, qu'elle prenne un moment sur le vif ou cadre soigneusement un portrait, imprime nécessairement une fixité au sujet représenté. Que ce soit pour Walter Benjamin (174-75) ou pour Barthes, "la nature de la Photographie, c'est la pose [...] cet instant, si bref fût-il, où une chose réelle s'est trouvée immobile devant l'œil " (1164). Laura Mulvey va même jusqu’à caractériser la photographie de pose "fossilisée " (18). Cette " rhétorique de la pose " est, en somme, assez simple : "Prendre une pose, c'est se présenter au regard d'autrui comme si on était déjà figé, immobile - c'est-à-dire déjà une image " (Owens, "Medusa Effect ", 198, ma traduction $)^{4}$. Chez Tournier et Orlan, le motif du suaire illustre cette problématique dans toutes ses suggestions.

Il suffit, par exemple, de réfléchir à la fonction du suaire, à savoir envelopper le cadavre, et de remarquer qu'il est question dans nos deux œuvres de Vésale, anatomiste de la Renaissance qui, parmi les premiers, pratiqua la dissection, et dont Véronique (162) tout autant qu'Orlan, qui, comme Vésale, fait de ses opérations chirurgicales un spectacle public (O’Bryan 39), s'inspirent dans leur travail. Or, Vésale accordait une grande importance à la mise en scène et présenta les cadavres de son De humani corporis fabrica (1543) dans une pose classique afin de leur prêter la " dignité » nécessaire (O’Bryan 49). Le ton est donné : le suaire fixe une pose.

3 Il convient néanmoins de signaler que l'œuvre d'Orlan est régulièrement étudiée sous l'angle de la performativité pour insister sur la fluidité de l'identité (voir Augsburg).

4 Par exemple, Colin Davis prend pour point de départ une analyse de Sarah Kofman, qui voit dans l'art un refus du deuil, le deuil étant selon Sigmund Freud l'acceptation de l'éphémère, et argumente que Tournier développe dans son œuvre et ses réflexions l'idée que la photographie « impose la stabilité au flux de l'identité » (35-36, ma traduction). 
Il n'est alors guère étonnant que Véronique et Orlan fassent mention de la qualité sculpturale du travail impliqué afin d'évoquer la fixité de l'image. Véronique veut donner à son modèle la "vérité [...] marmoréenne " (161) des cadavres et prononce un long discours sur son goût pour les statues. Orlan qualifie de "sculpture de chair » le travail chirurgical accompli sur son corps (Obrist 199) et explique son approche artistique, qui consiste à se servir de modèles existants pour en proposer une nouvelle vision, dans les termes suivants : "Citer, c'est me situer en figeant momentanément / ma propre réalité / et mon corps vivant / Pour lui infliger la froideur de marbre » (Wilson 15). De tels propos ne sont pas sans rappeler un essai capital de Louis Marin, dans lequel le « moment de la pétrification " de la Méduse ${ }^{5}$, que l'auteur nomme " moment sculptural » (136), symbolise la permanence que donne la peinture à l'éphémère, dès l'instant où il est capté sur la toile (139). La photographie serait alors un avatar moderne du geste du peintre et du mythe de la Méduse.

Tout comme une statue, le masque, autre élément dans l'œuvre de Véronique et d'Orlan, immobilise les traits et les mouvements, tout en cachant le visage, sur lequel il attire néanmoins l'attention. Ainsi, soumis à un régime draconien imposé par Véronique, afin qu’il devienne un modèle "photogénique» (156), Hector prend une apparence que remarque de suite le narrateur; il n'est plus qu'un "masque creusé, tout en pommettes, en menton et en orbites " (158), prêt, si l'on en croit la photographe, à devenir un chef-d'œuvre. Dans son étude de la carrière d'Orlan, C. Jill O’Bryan affirme " qu'avec les traces visibles de l'opération, le visage devient un masque. C'est ce phénomène qui rend le spectacle d'Orlan si perturbant : visuellement, son visage, que nous désirons percevoir en tant que 'visage', ne se lit plus comme 'visage', mais comme 'masque' " (105, ma traduction). En fait, Orlan, masquée, a fréquemment posé pour des photographes.

Dans le même ordre d'idée, le suaire est, somme toute, un écran, linge ou voile, qui cache le corps, mais aussi le révèle (le dévoile, pourrait-on dire) (Lemoine-Luccioni 77). Tout au long de sa carrière, Orlan a exploité le motif du voile dans ses réalisations, principalement sous la forme du drapé et du pli de l'habit de religieuse qu'elle revêt dans des séries telles que Sainte Orlan, dans lesquelles elle porte littéralement le voile. S’inspirant de la tradition baroque (voir Deleuze sur le baroque et le pli), elle affectionne plus particulièrement une sculpture de Giovanni Bernini, L'extase de sainte Thérèse et son drapé figé dans la pierre (Weintraub 78). Le pli de l'habit,

5 Le regard de la Méduse pétrifiait ses ennemis; elle fut elle-même changée en pierre lorsque Persée la décapita. 
dont on peut aisément deviner la souplesse, apparaît immobile sur les photos d'Orlan. Orlan le nomme "sculpture de pli "(Obrist 199); Eugènie Lemoine-Luccioni le décrit comme étant " pétrifié " (43), alors que Christine Buci-Glucksmann caractérise le baroque d'art de la pose (23). L'utilisation qu'Orlan fait du motif du voile n'est pas non plus étrangère à son engagement idéologique. En effet, cette dernière a explicitement présenté ses projets comme étant une réflexion féministe récusant le point de vue masculin en matière de représentation de la femme dans l'art et la société, y compris sa dimension religieuse. Elle déclare ainsi que son art " renverse le principe chrétien du verbe qui se fait chair au profit de la chair faite verbe" (Art charnel 2). Dans l'œuvre d'Orlan, le voile a la fonction d'un manifeste. Le vêtement est une véritable pièce à conviction soumise aux regards, porteuse du message de l'artiste. Pour sa part, la nouvelle de Tournier, si elle fait référence au suaire de Turin, évoque également le voile (ou sudario, linge qui sert à essuyer le visage; voir Bouloumié 49) que portait sainte Véronique. Or, ce voile a, de même, une valeur symbolique particulière; Véronique l'aurait posé sur le visage de Jésus, dont la trace serait restée figée sur le tissu. Dans les deux cas, le voile, pièce musée ou relique religieuse, est l'objet sur lequel se fixe le sens. Les suaires de Véronique et d'Orlan sont ainsi lourds d'un message, dont ils conservent les signes ostensibles.

En fait, faisant écho à l'ostentation du suaire de Turin, "Les suaires de Véronique " et les Saints-suaires sont, pourrait-on dire, ostentatoires, non pas en raison d'une mise en scène affectée et exagérée, mais plutôt parce qu'ils révèlent et exposent des signes, motifs et structures accompagnés d'abondantes déclarations de l'écrivain ou de l'artiste. De toute évidence, la dimension religieuse dans l'ensemble de l'œuvre de ces deux auteurs rend une telle ostentation d'autant plus sensible. Par exemple, Lorna Milne affirme que l'œuvre de Tournier recèle une opposition essentielle entre les images de la transfiguration et celles de la crucifixion (143). Quant à l'allusion à sainte Véronique dans la nouvelle, elle rappelle sans doute un commentaire de Barthes sur l'image photographique, dont il dit qu'elle est " résurrection ", car, comme le visage de Jésus sur le voile de la sainte, elle n'est pas créée directement par la main de l'homme (1167) (On appelle ce phénomène, qui est aussi celui invoqué pour décrire le suaire de Turin, achieropoitos). Orlan utilise, de même, la métaphore religieuse dans l'ensemble de ses réalisations. Titres et déclarations sont éloquents; on peut citer, entre autres, la série des Petits reliquaires ou celle de La réincarnation d'Orlan, de même que des phrases telles que "Ceci est mon corps... Ceci est mon logiciel..." (Art charnel 34). En fait, grâce à l'imagerie et aux 
rituels qu'elle exploite, Orlan "s'approprie l'énergie considérable " de la religion (Durand 208). Mise au service de la littérature et de l'art, la métaphore religieuse a une fonction d'exposition : elle exhibe les intentions ou vues de l'auteur.

L'image religieuse du suaire et son ostentation - et, de la sorte, toute pose photographique - ont ainsi pour effet de fasciner lecteurs et spectateurs, médusés, c'est-à-dire pétrifiés d'étonnement. En effet, les suaires de Véronique et d'Orlan ont été réalisés pour être exposés dans des galeries. L'immobilité des suaires tendus sur les murs et celle des statues, masques et voiles dont il est question dans nos deux œuvres, est alors celle des musées, des monuments et des objets de culte, c'est-à-dire celle qui attire les regards et l'intérêt, mais qui aussi, par voie de conséquence, suscite la parole, que ce soit pour critiquer ou admirer, voire adorer. Le suaire - et ceci s'applique donc à la pose photographique qu'il symbolise - incite à la parole : il demande explication; il suppose une histoire, il entraîne une réaction. Déployé, immobile, il invite à l'interprétation. En termes simples, le suaire est une trace à décrypter.

\section{Troublants suaires}

Or, c'est à ce stade que les signes se brouillent. Le décryptage se révèle paradoxal et n'indique aucune piste nette. L'empreinte du corps visible sur ces suaires est, c'est le moins qu'on puisse dire, troublante, tout comme la photographie, en faisant du sujet un objet, est troublante. Comment qualifier la présence humaine qu'on perçoit sur le suaire de Turin? Quel est le sujet de la photographie : celui qui pose, celui qui la prend ou celui qui la contemple? C'est précisément ce trouble du sujet, diversement manifesté, qu'explorent nos deux œuvres par le biais du motif du suaire. En premier lieu, il touche la question de savoir si la photographie est signe de présence ou d'absence. Sans doute l'immobilité que nos deux œuvres thématisent renforce-t-elle l'impression que la photographie, tout autant que le motif du suaire communiquent ou symbolisent la mortalité de tout être. Certes, il est largement admis que la photographie est hantée par la mort et qu'en conservant les traits et attitudes d'un individu sur la pellicule, elle est d'emblée un témoignage du passé et de ce qui n'est plus (Barthes 1163). Les scènes et portraits photographiés seront, après notre disparition, " reliques » de notre personne (Sontag 69). La photographie est donc l'art de l'absence absolue que signifie la mort. À cet effet, André Bazin note que « le Saint Suaire de Turin réalise la synthèse de la relique et de la photographie " (15). Selon ce critique, les arts plastiques ont pour 
« origine » " le 'complexe' de la momie », car la représentation dans l'art est refus de la mort : «Fixer artificiellement les apparences charnelles de l'être c'est l'arracher au fleuve de la durée : l'arrimer à la vie [...] La première statue égyptienne, c'est la momie de l'homme tanné et pétrifié dans le natron " (11). La photographie, continue-t-il, " embaume le temps" (15). Pour Benjamin, la photographie est l'art du " posthume " (174-75) et pour Susan Sontag "l'inventaire de la mortalité " (70); Barthes parle de " Mort plate" $(1174)^{6}$.

Néanmoins, de nombreux détails des "Suaires de Véronique " et des Saints-Suaires - de même que différents travaux critiques sur la photographie en général - suggèrent que la mort ne forme qu'un des grands thèmes qui occupent l'espace photographique. Ainsi, dans nos deux œuvres, l'accumulation de signes ostentatoires ne correspond pas uniquement à des rappels réitérés de l'absence, mais trahit aussi bel et bien une présence. Défiant tout entendement, le curieux phénomène qui se produit dans "Les suaires de Véronique " - un homme métamorphosé en suaire - matérialise ce paradoxe et crée littéralement un trouble insoluble dans la représentation du réel et la pensée de la mort. Que dire alors de la situation? Hector est-il présent ou absent? Dans la photographie, la présence et l'absence ne sont pas des notions opposées. Lattitude du narrateur est, à cet effet, exemplaire lorsqu'il visite la galerie exposant les suaires. Malgré le silence de la photographe et l'excès quasiment inimaginable de ses actions, il s'enquiert spontanément du jeune homme avec une pointe d'écœurement, car les suaires, somme toute objets abstraits, rendent toutefois Hector étrangement présent. Si le narrateur est choqué, c'est, d'une part, parce qu'il a pressenti d'emblée le sort réservé au jeune homme et, d'autre part, parce que ce dernier, en dépit des techniques radicales pratiquées par Véronique, n'a pas pu être réduit à une photographie. Ce suaire est paradoxalement, selon une expression de Barthes pour désigner l'impact de la photographie, le " certificat de présence " d'Hector (1169). La photographie, nous dit la nouvelle par le truchement du suaire, brouille les définitions usuelles de la vie et de la mort, de la présence et de l'absence. Dans l'abstraction et l'horreur des suaires, le narrateur perçoit Hector à ne pas s'y tromper. Mort, le jeune homme est encore véritablement présent. La photographie est, plus que tout autre art, un lien au réel : "Littéralement une émanation du référent " (1166), la

6 Le masque est, dans certains cas, un masque mortuaire modelant pour la postérité le visage du défunt. Il est alors un memento mori, tout comme la photographie (sur cet aspect de la photographie, voir Sontag 15). 
photographie, nous dit Barthes, est "la voie de la certitude " (1169; voir aussi Sontag 5), mais elle est aussi le doute porté sur le réel, le sujet absent recréé par la technologie, simple émanation.

Bien que la perspective adoptée soit autre, les Saints-Suaires d'Orlan ne suggèrent rien de bien différent. Sous les morceaux de gaze constitutifs du suaire, le visage d'Orlan est nettement reconnaissable. Plus même, l'immobilité de ses traits, la pose choisie, le regard fixé sur les spectateurs et les artifices sélectionnés (en particulier, le maquillage pour souligner les formes du visage) donnent non seulement l'impression du vivant, mais communiquent également la singularité du personnage : on pourrait presque dire que le visage d'Orlan crève le suaire. En outre, le regard direct est généralement pareil à une invitation au dialogue, du moins à un contact avec autrui, de même qu'il divulgue une identité. Certes, le sourire d'Orlan dans ses portraits et ses performances forme un trait distinctif auquel tout spectateur est sensible (Baqué 86). Autrement, dit, la mise en scène de l'absence et de la mort équivaut à l'étrange mise en scène d'une présence.

Derrière le suaire, Hector et Orlan sont "là " "Suaires de Véronique " 172). Le voile mortuaire, bien plus qu'il ne cache, les révèle. La photographie qui produit de tels suaires fait acte de présence; la trace humaine indélébile sur le linceul est signe de présence indélébile. La pose du jeune modèle et de l'artiste féministe face à l'appareil photo, certes cadavérique, affirme néanmoins leur individualité et l'intensité de leur présence. Nos symboliques suaires ont alors pour effet de déstabiliser ostensiblement l'opposition conceptuelle de l'absence et de la présence du sujet dans le portrait photographique, quitte à choquer le narrateur des "Suaires de Véronique " ou les spectateurs des Saints-suaires.

La deuxième manifestation du " trouble de civilisation " à signaler dans les deux ouvres a trait à la corporalité et va encore plus loin dans la déstabilisation du sujet. En effet, la peau, puisque c'est d'elle qu'il s'agit sur les suaires de Véronique et d'Orlan, est souvent considérée comme un site d'identité, contact de l'extérieur et de l'intérieur, limite et frontière du corps, lieu de l'apparence et des sensations. D'un point de vue psychanalytique, Didier Alzieu propose la notion de "Moi-Peau » pour désigner « une figuration dont le Moi de l'enfant se sert au cours des phases précoces de son développement pour se représenter lui-même comme Moi contenant les contenus psychiques, à partir de son expérience de la surface du corps » (39). Mais, chez Hector et Orlan devenus suaires, la question se pose différemment, car il n'y a dans les suaires ni intérieur ni extérieur; la peau n'est le contenant symbolique d'aucun contenu, puisqu'il ne reste de l'être que la peau. Par exemple, "réarrangeant " son corps et son identité 
grâce à la technologie médicale et multimédia, Orlan remet en cause « la notion du sujet cartésien, sujet qui est pure intériorité, son corps uniquement un contenant que la pensée transcende " (Jones 227, ma traduction); son art remémore "l'inexorable corporalité de l'être » (228). Ces peaux-suaires illustrent de la sorte que l'identité du sujet dans la pose photographique se singularise effectivement dans sa seule apparence physique.

En outre, plusieurs détails dans "Les suaires de Véronique » et dans les Saints-suaires suggèrent que de telles peaux-suaires, ou portraits photographiques, résultent d'un long travail de préparation et sont les produits d'efforts artistiques et d'une réflexion esthétique. Rien dans de tels portraits n'est laissé au hasard. Orlan et Véronique insistent fréquemment sur la nécessité du travail pour obtenir les meilleurs effets; "Les suaires de Véronique " et les propos d'Orlan sur son ouvre font mention de la maturation de l'expression, l'affinement d'une forme, la progression d'une pensée, la continuation d'une recherche ("Suaires de Véronique " 155, 157; Orlan, Art charnel). Curieusement, nos deux ouvres utilisent une même image pour caractériser les débuts du projet. Il s'agit de la coquille dans un des portraits d'Orlan datant des années 1960 (La naissance d'Orlan sans coquille), ainsi que dans une description d'Hector endormi dans une pièce où l'on "se serait cru à l'intérieur d'une coquille d'œuf " (164). Plus tard, le corps d'Hector, de même que celui d'Orlan, éclos d'une coquille, apprendront à trouver la pose qui sera conservée sur les suaires. En fait, l'empreinte du corps figée sur le suaire n'est pas sans rappeler que la pose, qu'elle soit choisie par le sujet ou imposée par l'artiste, évoque, dans quelque art que ce soit, un sujet constitué, formé, travaillé (voir Owens, "Posing »). Elle est l'achèvement d'une évolution depuis la coquille jusqu'au portrait fixé sur le tissu. Hector et Orlan, pures apparences fixées dans un portrait, sont certes de troublants sujets et les produits du " trouble de civilisation " qu'a introduit l'invention de la photographie dans la formation identitaire : nous avons appris à être objets, c'est-à-dire à poser pour nous-mêmes et pour autrui et à concevoir cette pose comme constituant notre identité (Lury 3).

Finalement, la troisième illustration du trouble identifié par Barthes et mis en scène par Tournier et Orlan rend compte des limites indécises entre le réel et le fictif dans toute photographie. Par le biais du motif du suaire, la nouvelle de Tournier et le projet d'Orlan illustrent en termes frappants qu'il n'existe en photographie ni lien brut au réel ni simple représentation d'un sujet; celui-ci est médiatisé, voilé, masqué, sculpté. Tournier choisit ainsi la littérature et ses réseaux d'images pour faire part de ses vues sur le 
réel de la photographie et l'enjeu de la pose. Quant à Orlan, en "substituant " son corps au langage, elle le transforme en message et en fiction (O’Bryan xiii). En fait, le thème de la peau incrustée dans un tissu n'est guère réaliste; il appartient, avant tout, à un imaginaire littéraire et artistique riche d'une longue tradition. Considérons, par exemple, qu’en dépit du martyre qu'elles évoquent ${ }^{7}$, nos deux ouvres donnent l'impression que la peau d'Hector après sa mort et celle d'Orlan sous la gaze demeurent intactes. La peau imprimée dans le suaire ne se dégrade pas, ne se dissout pas, ne subit pas les vicissitudes du temps. Or, le motif de la peau intacte, peau-signe, connait diverses variations littéraires. Par exemple, Peau d'âne dans le conte de Charles Perrault se vêt de la peau de l'animal pour fuir son père qui, séduit par sa jeunesse et sa beauté, souhaite l'épouser. Aussi longtemps qu'elle se couvre de cette peau, aucun homme ne s'intéresse à elle. Le prince ne tombe amoureux que lorsqu'elle quitte cet accoutrement. La peau de l'âne est symbole de virginité ${ }^{8}$. Dans La peau de chagrin d'Honoré de Balzac, Raphaël de Valentin échange son âme pour une "peau de chagrin » : tous ses désirs seront réalisés jusqu’à ce que cet étrange accessoire, que chaque désir rétrécit, disparaisse et signale la mort du héros. La peau de chagrin mesure la faiblesse morale de Raphaël, qui n'a pour autre objectif que l'assouvissement de ses désirs, et son abandon des valeurs qui ont formé sa jeunesse. Le portrait de Dorian Gray d'Oscar Wilde fait aussi du morcellement de la peau, dans ce cas son altération due au vieillissement, le point central de l'intrigue. Dorian Gray a conclu un pacte mystérieux grâce auquel il conservera l'apparence d'un homme jeune afin de profiter des plaisirs de la vie. En contrepartie, son portrait se flétrira un peu plus chaque fois qu'il commettra une faute morale. Dans ce marché, la peau intacte est signe d'intégrité. Le jour où les abus commis par le personnage dépassent le supportable, elle se fane et Dorian meurt.

Dans ces exemples, la peau jeune et intacte est signe d'une innocence idéale. Pourquoi ne pas imaginer qu'en exposant des suaires, Véronique et Orlan revisitent ce motif? Ce qui a plu initialement à Véronique lorsqu' elle a rencontré Hector n'était autre que sa jeunesse et son innocence; Hector était " un beau fruit juvénile " (157) d'une "patauderie un peu enfantine " (156). Personnage-nature, vierge de tous les apparats de la société, il lui donne l'idée de nouvelles techniques photographiques. Quant à Orlan, il se dégage de ses portraits et de ses films une indéniable impression

7 Tournier suggère que " prendre " la photographie d'un individu n'est guère différent de le "brûler en effigie" (Des clefs 103).

8 En 1990 paraît une série de photographies d'Orlan intitulées Peau d'àne. 
d'adolescence (Baqué 91). Son art célèbre l'innovation et la fraîcheur d'une révolte créatrice. Paradoxalement, le linge mortuaire porte la marque de la jeunesse. Le sujet de la photographie demeure alors figé dans un âge idéal et illusoire, celui du temps arrêté.

La photographie nous entraîne ainsi dans le mythe et la fiction. Art bouleversant, comme s'attachent à le montrer "Les suaires de Véronique " et les Saints-suaires, elle fait spectacle du paradoxe : la mort et la vie, l'absence et la présence, le corps et l'identité, le réel et l'imaginaire rendent toute photographie troublante et n'ont d'autres marques que celles, étranges et imprécises, fixées, telles celles du suaire, sur des clichés collectionnés dans des albums que nous nous plaisons à montrer à autrui pour raconter nos histoires.

\section{Ouvrages cités}

Anzieu, Didier. Le Moi-peau, Paris, Dunod, 1985.

Augsburg, Tanya. "Orlan's Performative Transformations of Subjectivity " in The Ends of Performance, dir. Peggy Phelan et Jill Lane, New York/Londres, New York UP, 1998, 285-314.

Baqué, Dominique. « Body-Orlan Becoming / Devenirs photographiques de l'Orlan-corps " in Orlan : Refigurations, Self-Hybridations, Dirs. Dominique Baqué, Marek Bartelik et Orlan, Paris, Al Dante, 2001, 76-95.

Barthes, Roland. La chambre claire : Note sur la photographie (1980) in Euvres complètes, tome III (1974-1980), dir. Eric Marty, Paris, Seuil, 1995, 1105-200.

Bazin, André. "Ontologie de l'image photographique " in Qu'est-ce que le cinéma? I - Ontologie et Langage, Paris, Cerf, 1958, 11-19.

Benjamin, Walter. Illuminations, trad. Harry Zohn, New York, Schocken, 1969.

Bouloumié, Arlette. Michel Tournier : Le roman mythologique suivi de Questions à Michel Tournier, Paris, Corti, 1988.

Buci-Glucksmann, Christine. Orlan, triomphe du baroque, Marseille, Images en Manœuvres, 2000.

Cros, Caroline; Laurent le Bon et Vivian Rehberg, dir., Orlan, trad. Deke Dusinberre, Paris, Flammarion, 2004.

Davis, Colin. "Art and the Refusal of Mourning : The Aesthetics of Michel Tournier », Paragraph 10, oct 1987, 29-44.

Deleuze, Gilles. Le pli, Paris, Minuit, 1988. 
Durand, Régis. "Texts for Orlan " in Orlan, dir. Caroline Caroline, Laurent le Bon et Vivian Rehberg, trad. Deke Dusinberre, Paris, Flammarion, 2004, 205-13.

Geimer, Peter. "Searching for Something. On Photographic Revelations » in Iconoclash, dirs. Bruno Latour et Peter Weibel, Karlsruhe/ Cambrigde, Mass., ZKM/MIT Press, 2002, 143-145.

Jean-Paul II. "Address of His Holiness Pope John Paul II " in Iconoclash, dir. Bruno Latour et Peter Weibel, Karlsruhe/Cambrigde, Mass., ZKM/The MIT P, 2002, 236-38.

Jones, Amelia. Body Art: Performing the Subject, Minneapolis, Univ. of Minnesota Press, 1998.

Kofman, Sarah. Mélancolie de l'art, Paris, Galilée, 1985.

Lemoine-Luccioni, Eugénie. La robe : Essai psychanalytique sur le vêtement, Paris, Seuil, 1984.

Lury, Celia. Prosthetic Culture : Photography, Memory and Identity, Londres/New York, Routledge, 1998.

Marin, Louis. To Destroy Painting, Trad. Mette Hjort, Chicago/Londres, niv.U of Chicago Press, 1995.

Milne, Lorna. L'Evangile selon Michel : La trinité initiatique dans l'œuvre de Michel Tournier, Amsterdam/Atlanta, Rodopi, 1994.

Mulvey, Laura. Death $24 x$ a Second: Stillness and the Moving Image, Londres, Reaktion, 2006.

Obrist, Hans Ulrich. "Orlan Interviewed » in Orlan. Dirs. Caroline Cros, Laurent le Bon et Vivian Rehberg, trad. Deke Dusinberre, Paris, Flammarion, 2004, 187-203.

O'Bryan, C. Jill. Carnal Art: Orlan's Refacing. Minneapolis/Londres, Univ. of Minnesota Press, 2005.

Orlan. De l'art charnel au baiser de l'artiste, Paris, Place, 1997.

Owens, Craig. "Posing » in Beyond Recognition : Representation, Power, and Culture, dir. Scott Bryson et al, Berkeley, Univ. of California Press, 1992, 201-17.

Owens, Craig. "The Medusa Effect, or the Specular Ruse " in Beyond Recognition: Representation, Power, and Culture, dir. Scott Bryson et al, Berkeley, Univ. of California Press, 1992, 191-200.

Rosello, Mireille. L'in-différence chez Michel Tournier, Paris, Corti, 1990. Sontag, Susan. On Photography, New York, Anchor \& Doubleday, 1977. Tournier, Michel. Des clefs et des serrures : Images et proses, Paris, Chêne/Hachette, 1979.

Tournier, Michel. "Les suaires de Véronique ", Le coq de bruyère, Paris, Gallimard, 1978, 151-172. 
Weintraub, Linda, Arthur Danto et Thomas McEvilley. «Orlan : SelfSanctification " in Art on the Edge and Over: Searching for Art's Meaning in Contemporary Society 1970s-1990s, Lichtfield, CT, Art Insights, 1996, 78-83.

Wilson, Sarah. "L'histoire d'O, sacrée et profane / L'histoire d'O, Sacred and Profane " in Orlan : Ceci est mon corps... Ceci est mon logiciel... I This Is My Body... This Is My Software... dir. Duncan McCorquodale, Londres, Black Dog, 1996, 7-17. 